PROCEEDINGS OF SYMPOSIUM:

THE ATHLETE'S ARM.

Held at the Wright Fleming Institute, St. Mary's Hospital, London W.C.2.

Sunday, February 20th, 1972.

\title{
THE ANATOMY OF THE ELBOW JOINT, WITH SPECIAL APPLICATION TO SPORTS INJURIES.
}

\author{
H. EVANS ROBSON, M.B., B.S. \\ Mountsorrel, Leicestershire.
}

The elbow joint is a hinge joint between the humerus above, and mainly the ulna below, but the radius forms a weaker and less stable articulation with the capitulum of the humerus. The range of movement is from about $30^{\circ}$ in flexion to $180^{\circ}$ in extension, and in some individuals even more than $180^{\circ}$ hyperextension may be found. In flexion, the forearm bones lie parallel to the humerus, but in extension there is some 50 to 100 external angulation, the "carrying angle", usually larger in females than in males. In pronation and supination, the head of the radius can rotate on the capitulum through some $180^{\circ}$, and the radial head also rotates at the superior radio-ulnar joint, in a lesions of the nerve roots, as in cervical spondylosis, or of the brachial plexus from to the ulnar, but surrounding the neck of the radius. The lateral (deltoid) ligament of the elbow runs from the lateral epicondyle of the humerus into the upper end of the ulnar, near the coronoid process and ulnar notch, but has its principal attachment into this annular ligament. (Fig. 1.)

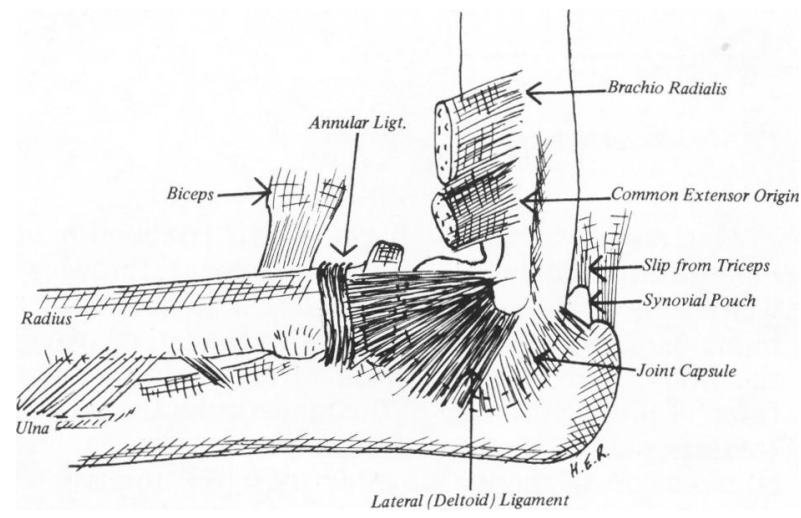

Fig. 1 Lateral Aspect of Left Elbow

Above the attachment of the deltoid ligament is the common origin of the long and short radial extensors of the wrist, and higher up the epicondyle the origin of the brachioradialis muscle. These three muscles form a mass on the outer aspect of the forearm separated from the other forearm muscles, anatomically and functionally.
Apart from their origins from the epicondyle, they receive an extensive origin from the deep fascia investing the forearm, mentioned in many of the older anatomical textbooks but in few modern ones. It is this superficial origin that sustains damage in repeated elbow extension with pronation of the forearm, such as occurs in tennis and badminton with overarm strokes, in the nondominant arm in golf, or more commonly in the do-ityourself enthusiast in painting the ceiling, or the publican in drawing pints.

The synovial membrane of the joint is extensive, especially on the posterior aspect of the joint. There is a pouch above the olecranon process that is normally pulled upwards by a slip of the triceps, that acts like the articularis genu muscle of the knee joint, and prevents the pouch from being trapped between the bony surfaces during extension. Sometimes a rapid extension movement of the elbow occurs before the pouch can be pulled out of the way, and this leads to acute synovitis and damages the joint capsule also. Rapid extension may also lead to avulsion fractures of the tip of the olecranon process.

The interosseous membrane, apart from giving a more extensive origin to the deep forearm muscles than can be provided by the bones alone, has a most important function in transmitting compression force on the hand from the strong lower end of the radius to the strong upper end of the ulna and the humero-ulnar joint, as neither the lower end of the ulna nor the upper end of the radius are strong enough or strongly enough attached to other joints, to give stability. The fibres of the membrane run downwards and medially, acting like tow-ropes to transmit their force. (Fig. 2.)

The medial side of the elbow joint is also prevalent to injury. The lateral ligament is strong, and reinforces the joint capsule. An anterior band runs from the medial epicondyle to the coronoid process of the ulna, and the posterior band from the epicondyle to the margin of the olecranon notch. A weaker structure, the middle band, runs between the coronoid and olecranon processes, has no attachment to the humerus, and forms a deep 

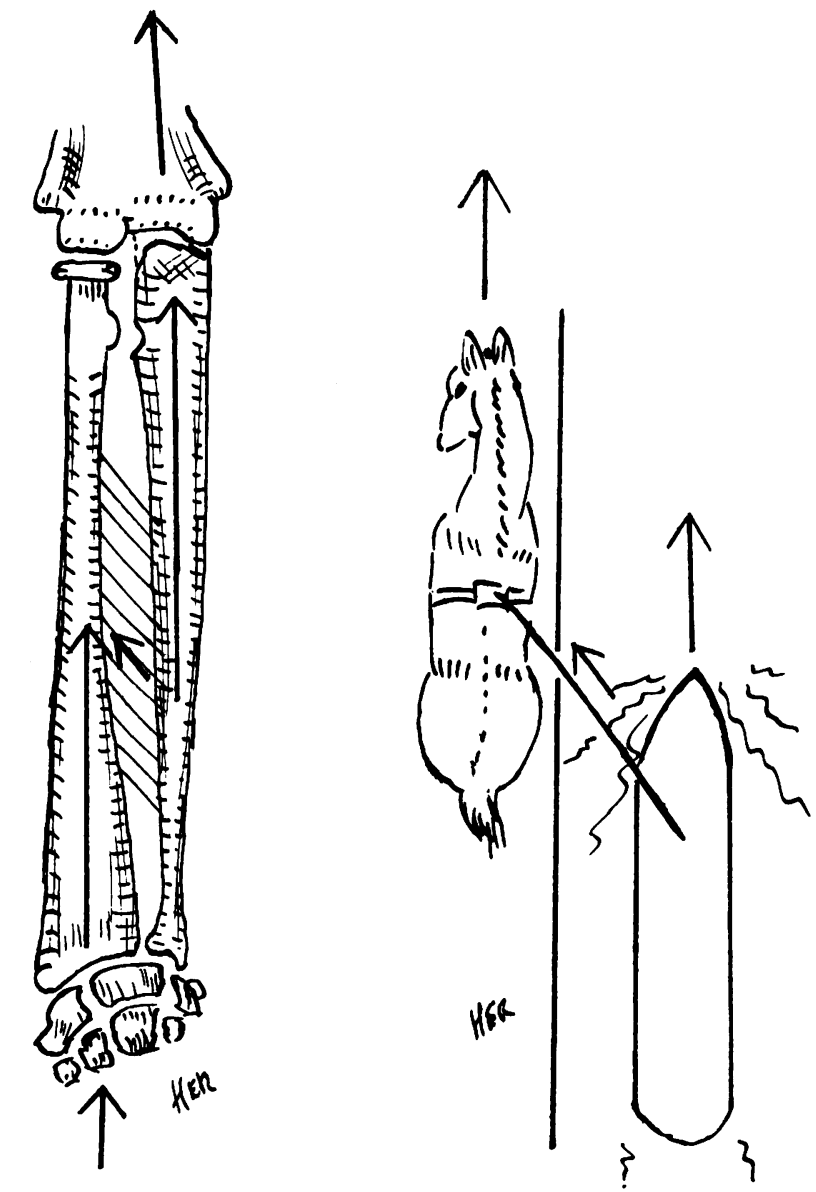

Fig. 2 The obliquity of the fibres of the Interosseous membrane enables compression on the hand to be transmitted from the strong lower end of the Radius to the strong upper end of the Ulnar. Then to the Humero-Ulnar joint in a similar manner to a horse pulling a barge on a parallel course by an oblique tow rope.

relation to the ulnar nerve. The ulnar nerve, supplying the small muscles of the hand and therefore essential for any action involving manual dexterity, lies in the upper arm behind the intermuscular septum, and gains the anterior compartment of the forearm by passing behind the medial epicondyle of the humerus, taking a straight line when the elbow is extended, but bending around the lower edge of the epicondyle during flexion. Injuries to any structure on the medial side of the elbow may lead to direct or indirect involvement of the ulnar nerve, with subsequent numbness or paresis of the hand. Complete tears of the nerve at this site are rare, but bruising and scarring of muscle or connective tissue, and fractures can cause pressure upon the nerve with impairment of its function, and may later necessitate transplant into the anterior compartment of the arm above the level of the epicondyle. It must not be forgotten that pain in the elbow region may be from lesions of the nerve roots, as in cervical spondylosis, or of the brachial plexus from for example a cervical rib, or even from postural faults and lack of tone in the trapezius muscle allowing the plexus to be stretched over the first rib.

From the medial epicondyle above the attachment of the ligament is the common flexor origin, but the flexor muscles of the forearm, like the radial extensors, also have an attachment to the deep investing fascia, and can sustain similar injuries, especially during activities that impose stress upon the medial aspect of the joint. This is one of the causes of "javelin elbow", which, like so many "specialist" conditions, may be due to one of several entirely different pathological lesions, each needing precise diagnosis and its specific treatment. (Fig. 3.)

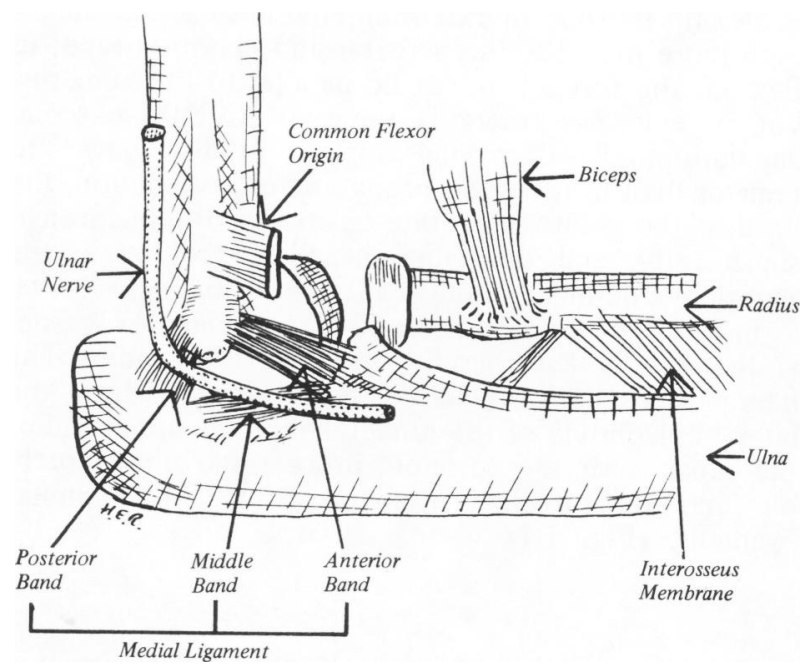

Fig. 3 Medial aspect of Left Elbow

Observation of many beginners to javelin throwing (and unfortunately on several prominent throwers as well) shows that a round-arm action is common; the humerus is abducted more or less in the lateral plane of the body, and the propulsion effort made by sudden forceful medial rotation of the humerus by the powerful rotators, subscapularis, pectoralis major, teres major and latissmus dorsi. the inertia effect of a long forearm with even the light weight of a javelin attached, imposes an enormous strain upon all the structures on the medial side of the elbow; - muscles, ligaments, and even the epicondyle itself. Accurate diagnosis is of prime importance. Obviously a fracture of the medial epicondyle, especially if the fragment is displaced into the joint, will require an entirely different approach to a ligament strain, a complete ligament tear, an ulnar neuritis, or a muscle injury. Once the condition has been treated, it is 
bound to recur unless the thrower changes his style completely, and uses extension of the elbow instead of medial rotation of the shoulder. These coaching points will be discussed in more detail, and with a great deal more authority, in the paper below by Colin Smith. (Fig. 4.)

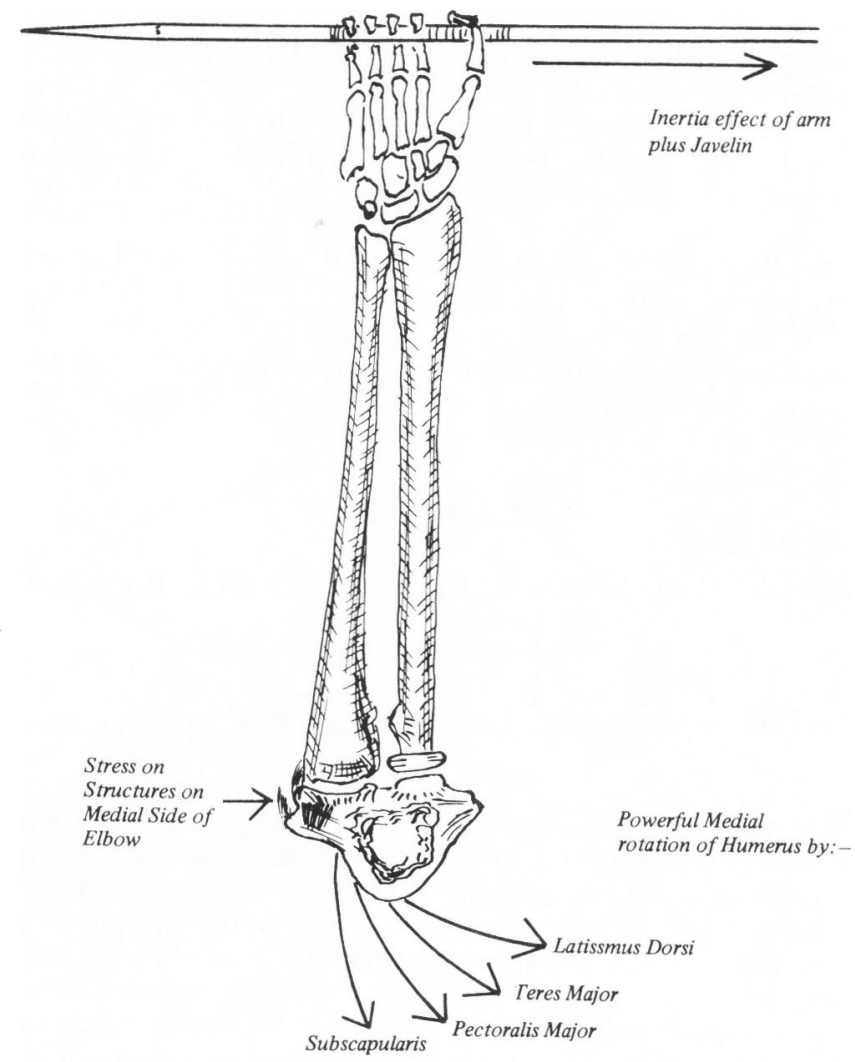

Fig. 4 Some Aetiological factors in "Javelin Elbow" when a round-arm action is used

BIOMECHANICS OF HITTING: BASIC USE OF THE ARM IN TENNIS

\section{SUZAN LIVINGSTONE}

\section{Beaconsfield School of Lawn Tennis}

The basic strokes used in the game of Lawn Tennis applied with or without a combination of spins are:-

1. Forehand Drive

2. Backhand Drive

3. Volleys (Forehand and Backhand)

4. Service

When coaching any of these basic strokes, one of the main difficulties encountered, as far as the arm is concerned, is teaching the player to have a complete racket control at all times during play.

Before this can be achieved, strong shoulder, upper arm and forearm muscles are necessary, as well as those of the wrist and hand. This is well shown by the majority of leading international players, particularly Rod Laver whose racket arm is very considerably more developed than the non playing arm, and when measured can be several inches larger in circumference.

During the time a player makes a stroke, it is desirable for the racket head to be kept higher than the level of the wrist. To do this when holding a racket weighing up to fourteen ounces, and when hitting a ball weighing approximately two ounces, considerable strength and control are required.

The three types of stroke consist of:- 